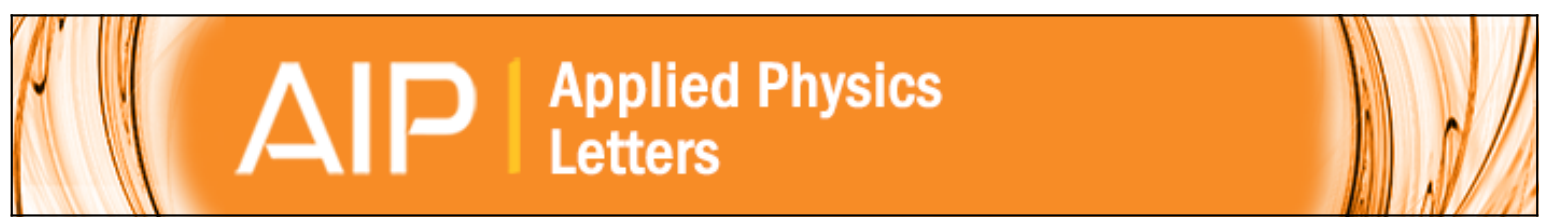

\title{
Out-of-plane current controlled switching of the fourfold degenerate state of a magnetic vortex in soft magnetic nanodots
}

Youn-Seok Choi, Myoung-Woo Yoo, Ki-Suk Lee, Young-Sang Yu, Hyunsung Jung, and Sang-Koog Kim

Citation: Applied Physics Letters 96, 072507 (2010); doi: 10.1063/1.3310017

View online: $\mathrm{http}: / / \mathrm{dx}$. doi.org/10.1063/1.3310017

View Table of Contents: http://scitation.aip.org/content/aip/journal/apl/96/7?ver=pdfcov

Published by the AIP Publishing

\section{Articles you may be interested in}

Radial-spin-wave-mode-assisted vortex-core magnetization reversals

Appl. Phys. Lett. 100, 172413 (2012); 10.1063/1.4705690

Understanding eigenfrequency shifts observed in vortex gyrotropic motions in a magnetic nanodot driven by spin-polarized out-of-plane dc current

Appl. Phys. Lett. 93, 182508 (2008); 10.1063/1.3012380

Reliable low-power control of ultrafast vortex-core switching with the selectivity in an array of vortex states by in-plane circular-rotational magnetic fields and spin-polarized currents

Appl. Phys. Lett. 92, 022509 (2008); 10.1063/1.2807274

Gyrotropic linear and nonlinear motions of a magnetic vortex in soft magnetic nanodots

Appl. Phys. Lett. 91, 132511 (2007); 10.1063/1.2783272

Electric-current-driven vortex-core reversal in soft magnetic nanodots

Appl. Phys. Lett. 91, 082506 (2007); 10.1063/1.2773748

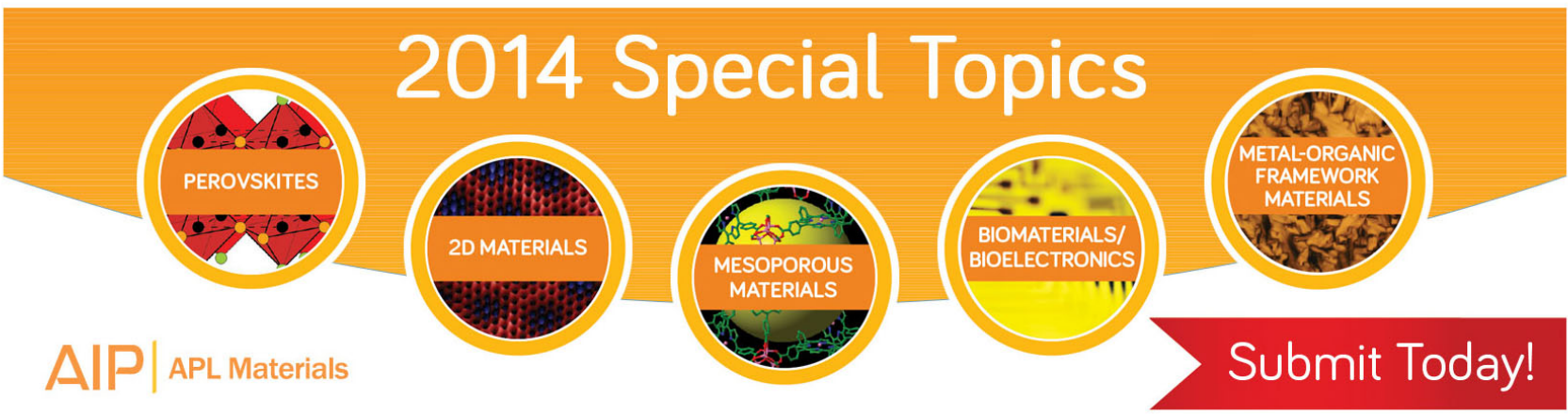




\title{
Out-of-plane current controlled switching of the fourfold degenerate state of a magnetic vortex in soft magnetic nanodots
}

\author{
Youn-Seok Choi, Myoung-Woo Yoo, Ki-Suk Lee, Young-Sang Yu, Hyunsung Jung, and \\ Sang-Koog $\mathrm{Kim}^{\text {a) }}$ \\ Research Center for Spin Dynamics \& Spin-Wave Devices, Nanospinics Laboratory, Research Institute of \\ Advanced Materials, Department of Materials Science and Engineering, Seoul National University, \\ Seoul 151-744, Republic of Korea
}

(Received 10 November 2009; accepted 16 January 2010; published online 17 February 2010)

\begin{abstract}
We report on an observation of transitions of the fourfold degenerate state of a magnetic vortex in soft magnetic nanodots by micromagnetic numerical calculations. The quaternary vortex states in patterned magnetic dots were found to be controllable by changing the density of out-of-plane dc or pulse currents applied to the dots. Each vortex state can be switched to any of the other states by applying different sequence combinations of individual single-step pulse currents. Each step pulse has a characteristic threshold current density and direction. This work offers a promising way for manipulating both the polarization and chirality of magnetic vortices. (C) 2010 American Institute of Physics. [doi:10.1063/1.3310017]
\end{abstract}

The unique magnetization configurations of magnetic vortices in the restricted geometries of magnetic nanodots of various shapes ${ }^{1,2}$ are of a growing interest. The vortex states are characterized by the combinations of an in-plane curling magnetization of either counter-clockwise $(c=+1)$ or clockwise $(c=-1)$ orientation (denoted by chirality $c$ ) along with an out-of-plane core magnetization of either upward $(p=+1)$ or downward $(p=-1)$ orientation (represented by polarization $p$ ).

In recent years, a dynamic phenomenon of ultrafast switching between the binary $p$ states has been observed by applications of not only linearly oscillating, ${ }^{3,4}$ circularly rotating $^{5-7}$ in-plane fields but also their pulse forms ${ }^{8}$ with lower-power consumption. In addition to such field-driven $p$ switching, in-plane ac (Refs. 9 and 10) and out-of-plane dc currents $^{11,12}$ have been reported to allow for the $p$ switching, as well.

Although there have been extensive studies on only $p$ switching, ${ }^{3-14}$ only $c$-switching. ${ }^{15,16}$ or switching of fluxclosure orientation in nanorings, the reliable control of transitions between the quaternary vortex states (fourfold degenerate states) has not been explored so far. In this letter, we present the results of micromagnetic numerical simulations of out-of-plane current driven switching of the fourfold degenerate vortex state. We also report a promising means of manipulating individual switching from each of the four states to any of the other states and back again, using different sequence combinations of characteristic single-step pulse currents, as found from this study.

In the present study, we used Permalloy (Py, $\mathrm{Ni}_{80} \mathrm{Fe}_{20}$ ) cylindrical dots of a radius $R=100 \mathrm{~nm}$ and different thicknesses, $L=10$ and $17 \mathrm{~nm}$, as shown in Fig. 1(a). The initial ground state was either $(p, c)=(+1,+1)$ or $(p, c)=(+1,-1)$. We numerically calculated the magnetization dynamic motions of individual unit cells (size: $2 \times 2 \times L \mathrm{~nm}^{3}$ Ref. 17) in the Py dots using the OOMMF code ${ }^{18}$ that employs the Landau-Liftshitz-Gilbert equation, ${ }^{19}$ including the so-called

\footnotetext{
${ }^{\text {a) }}$ Author to whom correspondence should be addressed. Electronic mail: sangkoog@snu.ac.kr.
}

Slonczewski spin-transfer torque (STT) ${ }^{20}$ The STT term is expressed as $T_{\mathrm{STT}}=\left(a_{\mathrm{STT}} / M_{S}\right) M \times\left(M \times \hat{m}_{P}\right) \quad$ with $a_{\mathrm{STT}}$ $=\frac{1}{2 \pi} h \gamma P j_{0} /\left(\mu_{0} 2 e M_{s} L\right)$, where $\hat{m}_{P}$ is the unit vector of spin polarization direction, $h$ the Plank's constant, $\gamma$ the gyromagnetic ratio, $P$ the degree of spin polarization, $j_{0}$ the current density, $\mu_{0}$ the vacuum permeability, $e$ the electron charge, and $M_{s}$ the saturation magnetization. In order to conduct the model study of spin-polarized out-of-plane current-driven vortex excitations in the free-standing dots, we assumed that the spin polarization points in the $-z$ direction, (i.e., $\left.S_{\text {pol }}=-1\right)$ along with $P=0.7$ [see Fig. 1(a)]. The current flow was in the $+z$ direction (denoted as $i_{\mathrm{p}}=+1$ ). The circumferential Oersted fields $(\mathrm{OHs})$ due to the current flow were taken into account using Biot-Savart's formulation. ${ }^{21}$ In such a free standing model of metallic materials, we did not con-

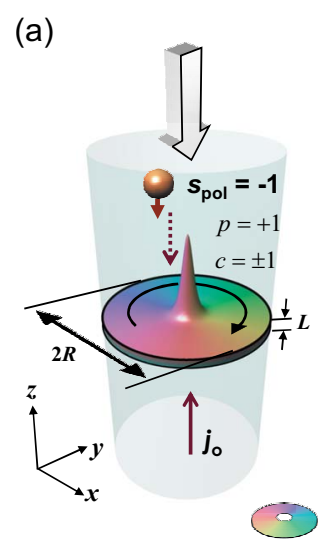

(b)
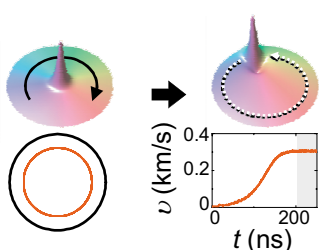

(c)

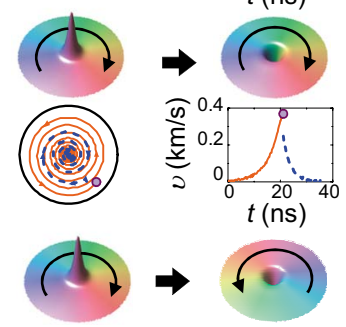

FIG. 1. (Color online) (a) Schematic illustration of the model geometry. The initial vortex states used are $(p, c)=(+1,+1)$ and $(+1,-1)$. The directions of the out-of-plane current and the spin polarization of electrons are denoted as $i_{\mathrm{p}}=+1$ and $S_{\mathrm{pol}}=-1$, respectively. The colors and the height of the surface correspond to the in-plane and out-of-plane components of the local magnetizations, respectively. (b), (c), and (d) represent simulation results on a vortex gyrotropic motion, only " $p$ " switching, and " $p$-PLUS-c" switching, respectively. The current densities used are $j_{0}=7.0 \times 10^{6}, 2.0 \times 10^{7}$, and $6.0 \times 10^{7} \mathrm{~A} / \mathrm{cm}^{2}$ for (b), (c), and (d), respectively. In (b) and (c) are also shown the orbital trajectories of motions of the up core (solid line) and the down core (dashed line) and their instantaneous speeds. 


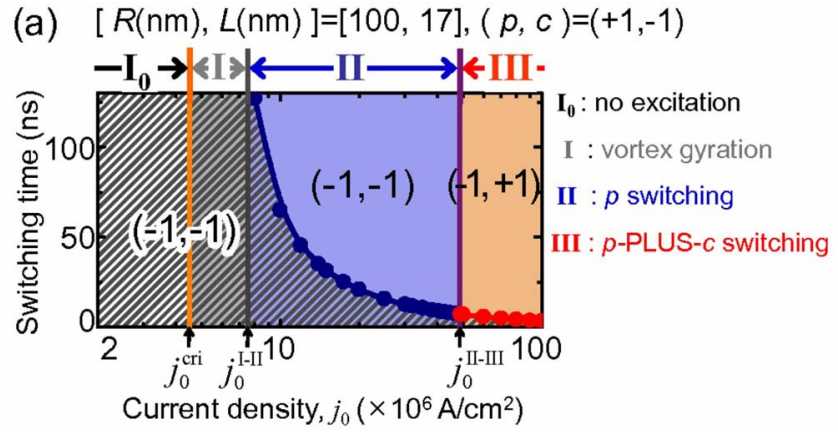

(b) $[100,17],(+1,+1)$ $[100,10],(+1,-1)$
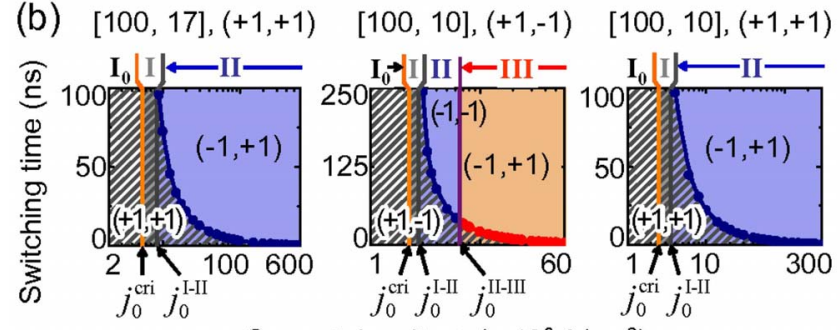

Current density, $j_{0}\left(\times 10^{6} \mathrm{~A} / \mathrm{cm}^{2}\right)$

FIG. 2. (Color online) Switching diagrams of no excitation, vortex gyration, " $p$ " and " $p$-PLUS- $c$ " switching with respect to $j_{0}$, as indicated by regimes, $\mathrm{I}_{0}$, I, II, and III, respectively. The closed symbols indicate the time required for each switching at a given value of $j_{0}$. The vertical lines represent the boundaries between the different vortex excitations, corresponding to the threshold $j_{0}$ values $\left(j_{0}^{\text {cri }}, j_{0}^{\mathrm{I}-\mathrm{II}}\right.$, and $\left.j_{0}^{\mathrm{II}-\mathrm{III}}\right)$ that distinguish each switching regime. The dot size and initial vortex state used are noted in each diagram. The hatched area corresponds to the initial ground state before " $p$ " and " $p$-PLUS-c" switching.

sider thermal heating by current flow because the temperature increase caused by the interface between an electrode and the nano-pillar is negligible. ${ }^{22}$ In real systems, however, the other interfaces of sufficiently large contact areas could yield considerable thermal heating in the cases of high current densities.

From simulation results on specific dot dimensions, e.g., $(R, L)=(100 \mathrm{~nm}, 17 \mathrm{~nm})$ and with the indicated initial state, $(p, c)=(+1,-1)$, three different characteristic dynamic behaviors are observed: a vortex gyrotropic motion, the switching of $p$ alone and the simultaneous switching of both $p$ and $c$, as represented in Figs. 1(b)-1(d), respectively. The dynamic behaviors from the $(p, c)=(+1,-1)$ state contrasted with different values of the current density $j_{0}$ applied, as shown in Fig. 1. Note that, with this initial vortex state, the circumferential $\mathrm{OH}$ orientation is antiparallel with the orientation of $c=-1$, and the orientation of $p=+1$ is parallel with the current direction, $i_{\mathrm{p}}=+1$. Figure 2 diagrams the distinct dynamic behaviors in different regimes of $j_{0}$. In regime $\mathrm{I}_{0}\left(j_{0}\right.$ below a certain critical density, $j^{\text {cri }}$ ) there is no further vortex excitation. In regime I $\left(j_{0}^{\text {cri }}<j_{0}<j_{0}^{\mathrm{IIII}}\right)$, we observed a typical low-frequency translation mode, the so-called vortex gyrotropic motion. ${ }^{21,23-25}$ In this regime, the vortex core exhibits a spirally rotating motion with a continuously increasing orbital radius, finally attaining the stationary motion of a constant orbital radius along with the eigenfrequency, as illustrated by the orbital trajectory of the vortex core motion and its speed [see Fig. 1(b)]. More detailed descriptions of regimes $\mathrm{I}_{0}$ and $\mathrm{I}$ are given in Ref. 24.

In contrast to the earlier $\mathrm{I}_{0}$ and I regimes, in regime II $\left(j_{0}^{\mathrm{IIII}}<j_{0}<j_{0}^{\mathrm{II}-\mathrm{III}}\right)$, only $p$ switching occurs, maintaining the initial state of $c=-1$ (without $c$-switching), as shown in Fig. 1(c), after the application of the dc current during the required $p$-switching time. The switching time, indicated by the solid circles and line in the diagrams, decreases with increasing $j_{0}$. For the lower $j_{0}$ values in regime II, the $p$-switching takes place via the creation and annihilation of a vortex-antivortex pair inside a given dot, according to the same mechanism as described in earlier studies. ${ }^{3,4,8}$ The velocity of the up core just before its switching was observed to be $330 \pm 37 \mathrm{~m} / \mathrm{s}$ [see Fig. 1(c)], which is the same as the critical velocity driven by oscillating in-plane and circular rotating fields (or currents). ${ }^{4}$ After the core orientation is reversed, the velocity of the down core decreases rapidly, and then there is no motion any more. Such out-of-plane dc current driven $p$ switching dynamics is analogous to a circularrotating-field driven core switching reported in Refs. 5-7. On the contrary, for the higher $j_{0}$ values in regime II, the $p$-switching mechanism differed from that observed in the lower $j_{0}$ region. Further investigation of the mechanisms is required. ${ }^{26}$

With further increases of $j_{0}$ across $j_{0}^{\text {II-III }}$, an additional vortex dynamic was found that represents the switching of both $p$ and $c$ with certain time intervals of the order of approximately nanoseconds (hereafter denoted as " $p$-PLUS- $c$ " switching). In this regime $\left(j_{0}>j_{0}^{\text {II-III }}\right)$, compared with the earlier regimes, the $j_{0}$ values are large, producing relatively high-strength $\mathrm{OHs}$, e.g., $1.25 \mathrm{kOe}$ at $j_{0}=2.0 \times 10^{8} \mathrm{~A} / \mathrm{cm}^{2}$ for $R=100 \mathrm{~nm}$ and $L=17 \mathrm{~nm}$. Thus, the high-strength $\mathrm{OH}$ assists such " $p$-PLUS- $c$ " switching via the reduction of the Zeeman energy through the additional $c$-switching from the antiparallel to the parallel orientation between the $c$-state and the given $\mathrm{OH}$. Note that such additional $c$ switching never happens for the initial state of $c \cdot i_{\mathrm{p}}=+1$ [see Fig. 2(b)]. However, the switching mechanism is not simple but different from the field driven magnetization reversals typically observed in ring-type elements. ${ }^{16}$ Further simulations reveal that the $c$ switching by only the $\mathrm{OH}$ (excluding STT effect) occurs above $j_{0}=1.8 \times 10^{8} \mathrm{~A} / \mathrm{cm}^{2}$, which value is six times greater than the threshold value, $j_{0}^{\text {II-III }}=0.3 \times 10^{8} \mathrm{~A} / \mathrm{cm}^{2}$ obtained considering both the STT and OH effects. ${ }^{26}$

Figure 2(b) shows additional switching diagrams for the different initial vortex states and dot size, as noted. General trends of those switching diagrams are the same for the case of $c=-1$, but there is no region III in the diagrams for the other case of $c=+1$. This is because the orientation of the $c$-state is parallel with that of the given $\mathrm{OH}$, as mentioned before.

On the basis of the results above, we propose a promising means by which each of the fourfold ground vortex states can be simply but reliably manipulated. In Fig. 3, we schematically illustrated ten different pulse sequences composed of single-, double- or triple-step pulses. Each current pulse is of either density value, $j_{0}^{\text {IIII }}<j_{0}<j_{0}^{\text {II-III }}$ or $j_{0}>j_{0}^{\text {II-III }}$, and either current direction, $+z$ or $-z$. The lengths of the step pulses are supposed to be at least longer than the switching times, $t_{\mathrm{p}}(10 \sim 250 \mathrm{~ns})$ and $t_{\mathrm{p}+\mathrm{c}}(<30 \mathrm{~ns})$, required for " $p$ " and " $p$-PLUS- $c$ " switchings, respectively. For example, for $p$ switching, single-step pulses with $t_{\mathrm{p}}$, (pulse types (1) and (2)), are necessary. Here, the current density and length of the step pulses were determined by the initial vortex state and the dot dimensions. Contrastingly, $c$-state switching can be achieved only by combining the two different processes of " $p$-PLLUS- $c$ " and " $p$ " switching, in order to return the $p$-state 


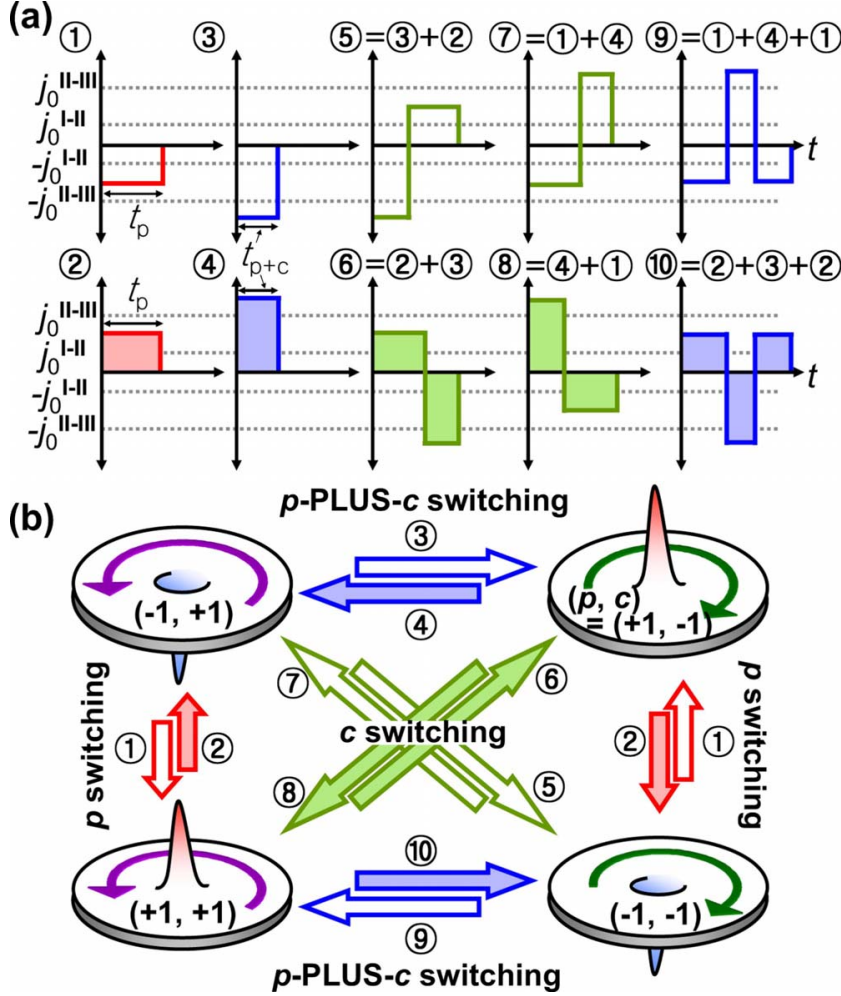

FIG. 3. (Color online) (a) Current pulse sequences (comprised of single, double, or triple pulses) required for individual switching between each pair of the quadruple vortex states shown in (b).

to its original state, since $c$-switching never occurs alone, but occurs along with $p$ switching. The pulse types, (5), (6), (7), and 8 effect $c$-switching. The pulse sequences for " $p$-PLUS- $c$ " switching are relatively complex: the singlestep pulses of (3) and (4) are required for switching between $(p, c)=(+1,-1)$, and $(-1,+1)$, but for switching between $(p, c)=(+1,+1)$ and $(-1,-1)$, the triple-step pulses of (9) and (10) are necessary. The reason is that the latter switching can occur only through the serial processes of switching from the given $(-1,-1)$ state to $(+1,-1)$ and $(-1,+1)$, and then to $(+1,+1)$, or vice versa. Thus, the pulse sequences (9) and (10), are the results of the combinations of (1) +(4)+(1) and (2)+(3)+(2), respectively. Consequently, each vortex state can be switched to any of the other states directly through two different processes of the " $p$ " and " $p$-PLUS- $c$ " switching or their combinations, by one of the pulse sequences ${ }^{27}$ indicated by the arrows and the numbers noted in Fig. 3 .

In summary, we have studied transitions of the fourfold degenerate state (both polarization and chirality) of a vortex in soft magnetic dots, manipulated by changing the density and direction of out-of-plane dc or pulse currents applied to the dots. It is proposed that individual switchings from each vortex state to any of the other states are controllable with the different sequences of four characteristic single-step pulses found from this study. This work provides a means of controlling the full degree of freedom of the fourfold degenerate state of a magnetic vortex in confined magnetic dots.
This work was supported by Basic Science Research Program through the National Research Foundation of Korea (NRF) funded by the Ministry of Education, Science and Technology (Grant No. 20090063589).

${ }^{1}$ T. Shinjo, T. Okuno, R. Hassdorf, K. Shigeto, and T. Ono, Science 289 930 (2000); A. Wachowiak, J. Wiebe, M. Bode, O. Pietzsch, M. Morgenstern, and R. Wiesendanger, ibid. 298, 577 (2002).

${ }^{2}$ L. Thomas, C. Rettner, M. Hayashi, M. G. Samant, S. S. P. Parkin, A Doran, and A. Scholl, Appl. Phys. Lett. 87, 262501 (2005); J.-Y. Lee, K.-S. Lee, S. Choi, K. Y. Guslienko, and S.-K. Kim, Phys. Rev. B 76, 184408 (2007).

${ }^{3}$ B. Van Waeyenberge, A. Puzic, H. Stoll, K. W. Chou, T. Tyliszczak, R. Hertel, M. Fähnle, H. Brückl, K. Rott, G. Reiss, I. Neudecker, D. Weiss, C. H. Back, and G. Schütz, Nature (London) 444, 461 (2006).

${ }^{4}$ K.-S. Lee, K. Y. Guslienko, J.-Y. Lee, and S.-K. Kim, Phys. Rev. B 76, 174410 (2007); K. Y. Guslienko, K.-S. Lee, and S.-K. Kim, Phys. Rev. Lett. 100, 027203 (2008).

${ }^{5}$ S.-K. Kim, K.-S. Lee, Y.-S. Yu, and Y.-S. Choi, Appl. Phys. Lett. 92, 022509 (2008).

${ }^{6}$ M. Curcic, B. Van Waeyenberge, A. Vansteenkiste, M. Weigand, V. Sackmann, H. Stoll, M. Fahnle, T. Tyliszczak, G. Woltersdorf, C. H. Back, and G. Schutz, Phys. Rev. Lett. 101, 197204 (2008).

${ }^{7}$ K.-S. Lee, S.-K. Kim, Y.-S. Yu, Y.-S. Choi, K. Y., Guslienko, H. Jung, and P. Fischer, Phys. Rev. Lett. 101, 267206 (2008)

${ }^{8}$ R. Hertel, S. Gliga, M. Fähnle, and C. M. Schneider, Phys. Rev. Lett. 98, 117201 (2007).

${ }^{9}$ K. Yamada, S. Kasai, Y. Nakatani, K. Kobayashi, H. Kohno, A. Thiaville, and T. Ono, Nature Mater. 6, 270 (2007).

${ }^{10}$ S.-K. Kim, Y.-S. Choi, K.-S. Lee, K. Y. Guslienko, and D.-E. Jeong, Appl. Phys. Lett. 91, 082506 (2007).

${ }^{11}$ J.-G. Caputo, Y. Gaididei, F. G. Mertens, and D. D. Sheka, Phys. Rev. Lett. 98, 056604 (2007).

${ }^{12}$ D. D. Sheka, Y. Gaididei, and F. G. Mertens, Appl. Phys. Lett. 91, 082509 (2007); Y. Liu, H. He, and Z. Zhang, ibid. 91, 242501 (2007).

${ }^{13}$ S.-K. Kim, K.-S. Lee, Y.-S. Choi, and Y.-S. Yu, IEEE Trans. Magn. 44 3071 (2008)

${ }^{14}$ S. Bohlens, B. Kruger, A. Drews, M. Bolte, G. Meier, and D. Pfannkuche, Appl. Phys. Lett. 93, 142508 (2008).

${ }^{15}$ B. C. Choi, J. Rudge, E. Girgis, J. Kolthammer, Y. K. Hong, and A. Lyle, Appl. Phys. Lett. 91, 022501 (2007).

${ }^{16}$ T. Yang, M. Hara, A. Hirohata, T. Kimura, and Y. Otani, Appl. Phys. Lett. 90, 022504 (2007); M. T. Moneck and J.-G. Zhu, IEEE Trans. Magn. 44, 2500 (2008)

${ }^{17}$ We used the dot thickness $L$ for the cell dimension along the $z$ direction The material parameters corresponding to Py were as follows: the saturation magnetization $M_{s}=8.6 \times 10^{5} \mathrm{~A} / \mathrm{m}$, the exchange stiffness $A_{\mathrm{ex}}=1.3$ $\times 10^{-11} \mathrm{~J} / \mathrm{m}$, the damping constant $\alpha=0.01$, and the gyromagnetic ratio $\gamma=2.21 \times 10^{5} \mathrm{~m} / \mathrm{As}$, with a zero magnetocrystalline anisotropy.

${ }^{18}$ The version of the OOMMF code used is $1.2 \mathrm{a} 4$. See http://math.nist.gov/ oommf.

${ }^{19}$ L. D. Landau and E. M. Lifshitz, Phys. Z. Sowjetunion 8, 153 (1935); T. L. Gilbert, Phys. Rev. 100, 1243 (1955).

${ }^{20}$ J. C. Slonczewski, J. Magn. Magn. Mater. 159, L1 (1996).

${ }^{21}$ Y.-S. Choi, S.-K. Kim, K.-S. Lee, and Y.-S. Yu, Appl. Phys. Lett. 93, 182508 (2008).

${ }^{22}$ C.-Y. You, S.-S. Ha, and H.-W. Lee, J. Magn. Magn. Mater. 321, 3589 (2009); S.-S. Ha and C.-Y. You, Phys. Status Solidi A 204, 3966 (2007).

${ }^{23}$ B. A. Ivanov and C. E. Zaspel, Phys. Rev. Lett. 99, 247208 (2007).

${ }^{24}$ Y.-S. Choi, K.-S. Lee, and S.-K. Kim, Phys. Rev. B 79, 184424 (2009).

${ }^{25}$ K. Yu. Guslienko, B. A. Ivanov, V. Novosad, Y. Otani, H. Shima, and K. Fukamichi, J. Appl. Phys. 91, 8037 (2002).

${ }^{26}$ K.-S. Lee, M.-W. Yoo, and S.-K. Kim (unpublished).

${ }^{27}$ All of the simulation results shown in this paper are specific to the simulation conditions described in Fig. 1(a) and the text. The ten types of pulse sequences shown in Fig. 3 were also demonstrated for the " $p$ " and " $p$-PLUS-c" switchings for another conditions while holding $p \bullet S_{\mathrm{pol}}=-1$. With the other conditions for $p \cdot S_{\mathrm{pol}}=+1$, there were neither gyrotropic motion and nor any switching. 